\section{Statins improve endothelial function in patients with lacunar infarctions}

Accumulating evidence indicates that statin therapy might protect against stroke. It is postulated that statins might exert this protective effect through the improvement of endothelial homoeostasis by increasing the bioavailability of nitric oxide. Using cerebrovascular reactivity to L-arginine as a reflection of cerebral endothelial function, and flow-mediated dilatation to evaluate systemic endothelial function, PretnarOblak et al. carried out a small study to examine the effect of statin treatment on patients with lacunar infarctions (LI), who are thought to have a primary endothelial impairment.

The study comprised 18 patients with LI, 20 age-matched individuals showing similar cardiovascular risk factors (CRFs) to those exhibited by the LI group, and 19 age-matched healthy individuals. Cerebral and systemic endothelial function were measured in all participants at study start and 3 months later; between these time points, the patients with $\mathrm{LI}$ and those with CRF received daily atorvastatin treatment $(40 \mathrm{mg}$ ).

At baseline, L-arginine reactivity was lower in patients with $\mathrm{LI}$ and in those with CRFs than in healthy controls ( $P \leq 0.05$ for both). Following atorvastatin treatment, L-arginine reactivity improved significantly over baseline in both the LI group and the CRF group ( $P \leq 0.01$ for both). Flowmediated dilatation also improved in both of these groups following atorvastatin treatment; however, the improvement was more pronounced in those with LI, perhaps indicating a systemic predilection for endothelial damage. The authors conclude that a large double-blind study is warranted to further confirm the beneficial effect of statins on endothelial function.

Original article Pretnar-Oblak J et al. (2006) Influence of atorvastatin treatment on $\mathrm{L}$-arginine cerebrovascular reactivity and flow-mediated dilatation in patients with lacunar infarctions. Stroke 37: 2540-2545

\section{New evidence-based guidelines for the management of transient ischemic attack}

Transient ischemic attack (TIA) is often a precursor to major ischemic stroke, and appropriate management of the condition is, therefore, highly important. Currently, considerable variation exists in how the condition is managed, and the majority of published treatment guidelines are several years old. Newly updated evidencebased, unbiased guidelines sponsored by the National Stroke Association were recently reported in Annals of Neurology, which underline the need for urgent evaluation and treatment of individuals who experience TIA.

Fifteen selected experts carried out a systematic literature review and identified 257 published sets of guidelines on TIA, 13 of which met the entry criteria for their study. The recommendations derived from the guidelines were independently assigned quality ratings, and those with the highest ratings were edited through a series of questionnaires until a consensus was reached on the wording of the new recommendations. The final 53 recommendations were then rated on the basis of their level of evidence and quality.

Five major categories of recommendation are included in the new guidelines: initial management; evaluation; medical treatment; surgical treatment; and risk factor management. At the conclusion of the study process, the experts agreed that all of the recommendations could also be applied to patients who had recently experienced a minor ischemic stroke.

The authors conclude that the new guidelines should receive wide distribution amongst clinicians caring for patients with TIAs, not only through brochures and pocket cards, but also through a website that has been created, which will be updated as required.

Original article Johnston SC et al. (2006) National Stroke Association guidelines for the management of transient ischemic attacks. Ann Neurol 60: 301-313

\section{Increased cortical excitability could induce epileptic seizures after sleep deprivation}

Sleep deprivation is known to activate seizures in most types of epilepsy, but the pathophysiology underlying this effect is unclear. In a recent study, researchers used transcranial magnetic stimulation (TMS) to investigate the effects of sleep deprivation on cortical excitability in patients with epilepsy.

Badawy et al. recruited 15 patients with idiopathic generalized epilepsy (IGE) and 15 patients with focal epilepsy; all patients were newly diagnosed and had not been receiving treatment for their condition. In addition, 13 healthy control 